\title{
Heteroclinic Solutions for Nonautonomous EFK Equations
}

\author{
Y. L. Yeun ${ }^{1,2}$ \\ ${ }^{1}$ School of Mathematics and Systems Science, Beijing University of Aeronautics and Astronautics, Beijing 100191, China \\ ${ }^{2}$ School of Mathematical Sciences, Peking University, Beijing 100875, China
}

Correspondence should be addressed to Y. L. Yeun; ruanyl@math.pku.edu.cn

Received 30 August 2012; Accepted 12 February 2013

Academic Editor: Nikolaos Papageorgiou

Copyright (C) 2013 Y. L. Yeun. This is an open access article distributed under the Creative Commons Attribution License, which permits unrestricted use, distribution, and reproduction in any medium, provided the original work is properly cited.

We explore the nonautonomous fourth-order differential equation which has important applications in materials science. By variational approach, we find heteroclinic solutions of the equation. The conditions on the potential function $V(t, u)$ are mild enough to include a broad class of equations. We also consider a separate case where $V(t, u)$ is periodic in $t$.

\section{Introduction}

The goal of this paper is to study the nonautonomous extended Fisher-Kolmogorov (EFK) equation

$$
q^{\prime \prime \prime \prime}(t)-q^{\prime \prime}(t)+V_{q}(t, q)=0,
$$

where $V(t, q)$ is a time-dependent potential function and $V_{q}$ denotes the partial derivative with respect to the second variable $q$.

Fourth-order differential equations, which often appear in nonlinear elasticity, fluid mechanics, and relating physical problems, have received growing attention from researchers. For example, Peletier and Troy [1-4] studied the EFK equation with odd nonlinearity

$$
\mu q^{\prime \prime \prime \prime}-\lambda q^{\prime \prime}-q+q^{3}=0, \quad(\mu, \lambda>0),
$$

mainly using shooting argument and variational method. They found the existence of periodic, heteroclinic, and homoclinic solutions and proved the existence of chaotic solutions oscillating between 1 and -1 with all critical points being either minima or maxima. They also explored the boundedness, monotonicity, and other quantitative properties of solutions. Part of Peletier and Troy's work was generalized by Kalies and VanderVorst [5]; they considered the EFK equation with general nonlinear term

$$
\mu q^{\prime \prime \prime \prime}-\lambda q^{\prime \prime}+V^{\prime}(q)=0, \quad(\mu, \lambda>0),
$$

where $V(q)$ is supposed to have symmetric wells with equal depth and grow superquadratically. Then, they investigated the multitransition structure of heteroclinic and homoclinic solutions. Primary examples are

$$
V_{1}(q)=\frac{1}{4}\left(q^{2}-1\right)^{2}, \quad V_{2}(q)=\frac{2}{\pi^{2}}(1+\cos \pi q) .
$$

The symmetric property of $V(q)$ is rather restrictive, and many physical problems do not satisfy such conditions. Later, Kalies et al. $[6,7]$ obtained heteroclinic and homoclinic solutions connecting saddle-focus equilibria, and the potential function $V(q)$ is assumed to grow superquadratically and has at least two nondegenerate global minima, not necessarily symmetric. In case of (3) with $V(q)=V_{1}(q)$, the presence of saddle-focus equilibria amounts to the requiring of $4 \mu>$ $\lambda^{2} / V_{1}^{\prime \prime}( \pm 1)$. Periodic and chaotic solutions of (3) are also explored. These results were generalized in recent works [8, 9], where the author obtained heteroclinic solutions for (3) under very mild conditions of $V$; in particular, they do not assume that $V$ is symmetric or that saddle-focus equilibria are present.

We have just mentioned a few works that are closely related to our results. A good summary of typical researches in fourth-order differential equations can be found in [10].

In this paper we step forward to study heteroclinic solutions of the nonautonomous EFK equation (1). We are inspired by Yeun [9], Rabinowitz [11, 12], and Izydorek and Janczewska [13]. However, we are emphasizing that the argument of $[9,11]$ relies on the fact that the equation is 
autonomous; therefore, the method there cannot be reproduced here to tackle the time-dependent version (1) which is no longer autonomous. The nonautonomous case necessitates careful analysis. Another point should be made is that we are working on the $\left(q, q^{\prime}\right)$ phase plane; this is essentially different from $[11,13]$. In our argument, we also benefit from analysis of $[3,4]$ and comments of [5].

In spite of its practical significance [14], there has been few researches on (1) with time-dependent potentials; the paper seems to be the first attempt in the direction and the methods here can be applied to similar equations.

\section{Heteroclinic Solutions}

By a heteroclinic solution connecting $a_{-}$and $a_{+}$, we mean a solution $q(t)$ verifying

$$
\begin{aligned}
& \left(q(t), q^{\prime}(t), q^{\prime \prime}(t), q^{\prime \prime \prime}(t)\right) \longrightarrow\left(a_{+}, 0,0,0\right), \quad t \longrightarrow+\infty \\
& \left(q(t), q^{\prime}(t), q^{\prime \prime}(t), q^{\prime \prime \prime}(t)\right) \longrightarrow\left(a_{-}, 0,0,0\right), \quad t \longrightarrow-\infty
\end{aligned}
$$

Throughout the paper, let $B_{\varepsilon}(C)$ denote the neighborhood of a set $C \subset \mathbb{R}^{n}$ defined as below

$$
B_{\varepsilon}(C)=\left\{w \in \mathbb{R}^{n}\left|\inf _{v \in C}\right| w-v \mid<\varepsilon\right\} .
$$

We will make the following assumptions:

$\left(A_{1}\right) V(t, q) \in C^{2}(\mathbb{R} \times \mathbb{R}, \mathbb{R}), V \geqslant 0 ;$

$\left(A_{2}\right)$ let $\mathscr{V}$ be a set which has at least two points and we assume the points in $\mathscr{V}$ do not collapse together,

$$
\gamma \equiv \frac{1}{3} \inf \{|x-y| \mid x \neq y \in \mathscr{V}\}>0
$$

$\left(A_{3}\right)$ one of the following is satisfied:

(a) there is $\varepsilon^{*} \in(0, \gamma]$, such that $\hat{\sigma}_{\varepsilon} \inf _{z \notin B_{\varepsilon}(\mathscr{V}), t \in \mathbb{R}}$ $V(t, z)>0$ for any $\varepsilon \in\left(0, \varepsilon^{*}\right]$;

(b) $\liminf |z| \rightarrow \infty|z| V(t, z)>0$ uniformly for $t \in \mathbb{R}$.

$\left(A_{4}\right) \lim _{|t| \rightarrow+\infty} V(t, z)=+\infty$ uniformly for $z$ in any compact subsets of $\mathbb{R} \backslash \mathscr{V}$.

$\left(A_{5}\right)$ For each $z \in \mathscr{V}$,

$$
\int_{-\infty}^{\infty} V(t, z) d t<\gamma \sigma_{\gamma}^{1 / 2}
$$

where $\sigma_{\gamma}$ is defined in $\left(A_{3}\right)$ with $\varepsilon=\gamma$.

Theorem 1. Let $V(t, q)$ satisfy $\left(A_{1}\right)-\left(A_{4}\right)$. Then for every $z \in$ $\mathscr{V}$, there is a pair of heteroclinic solutions of (1), one emanating from $z$ and the other terminating at $z$.

Remark 2. To be noted, we do not assume $V$ has wells of equal depth. By the assumptions of the theorem, we necessarily have $\{z \in \mathbb{R} \mid V(t, z)=0$ for all $t\} \subset \mathscr{V}$. However, $\mathscr{V}$ may contain other points which are not global minima of $V(t, u)$ but still verify $\left(A_{5}\right)$. In the special case $\mathscr{V}=\{z \in \mathbb{R} \mid V(t, z)=$ 0 for all $t\},\left(A_{5}\right)$ is automatically satisfied.

Notation. Define $\widetilde{\mathscr{V}}=\left\{(z, 0) \in \mathbb{R}^{2} \mid z \in \mathscr{V}\right\}$. For an element $(z, 0)$ in $\widetilde{\mathscr{V}}$, we denote it by $\widetilde{z}=(z, 0)$ for brevity. Whence, without further notice, we will use $z$ and $\widetilde{z}$ where it is appropriate to denote element in $\mathscr{V}$ and its counterpart in $\widetilde{\mathscr{V}}$.

We will obtain heteroclinic solutions of (1) by minimizing the energy functional $I(\cdot)$ on an appropriate subset

$$
I(q)=\int_{-\infty}^{\infty}\left\{\frac{1}{2}\left(q^{\prime \prime}\right)^{2}+\frac{1}{2}\left(q^{\prime}\right)^{2}+V(t, q)\right\} d t .
$$

Denote the energy on a bounded interval $[r, s]$ by

$$
I_{r, s}(q)=\int_{r}^{s}\left\{\frac{1}{2}\left(q^{\prime \prime}\right)^{2}+\frac{1}{2}\left(q^{\prime}\right)^{2}+V(t, q)\right\} d t .
$$

We will work on the space

$$
E=\left\{q \in W_{\mathrm{loc}}^{2,2}(\mathbb{R}, \mathbb{R}) \mid \int_{-\infty}^{\infty}\left(\left|q^{\prime \prime}\right|^{2}+\left|q^{\prime}\right|^{2}\right) d t<\infty\right\},
$$

equipped with the norm

$$
\|q\|^{2}=\int_{-\infty}^{\infty}\left(\left|q^{\prime \prime}\right|^{2}+\left|q^{\prime}\right|^{2}\right) d t+|q(0)|^{2}
$$

It is readily seen that $(E,\|\cdot\|)$ is a Hilbert space and $q \in E$ implies $q \in C^{1}(\mathbb{R})$.

Let $\widetilde{\vartheta} \in \widetilde{\mathscr{V}} \backslash\{\widetilde{0}\}, \varepsilon \in(0,1)$. Denote by $\Gamma_{\varepsilon}(\vartheta)$ the set of $q \in E$ verifying

(i) $\lim _{t \rightarrow-\infty} q(t)=0, \lim _{t \rightarrow+\infty} q(t)=\vartheta$;

(ii) $\left(q(t), q^{\prime}(t)\right) \notin B_{\varepsilon}(\widetilde{\mathscr{V}} \backslash\{\widetilde{0}, \widetilde{\mathcal{\vartheta}}\})$, for all $t \in \mathbb{R}$.

That is, $\Gamma_{\varepsilon}(\vartheta)$ consists of functions which join 0 and $\vartheta$, and at least keep a distance of $\varepsilon$ to the set $\widetilde{\mathscr{V}} \backslash\{\widetilde{0}, \widetilde{\vartheta}\}$. Clearly, $\Gamma_{\varepsilon}(\vartheta)$ is not empty, we may consider the minimization problem

$$
c_{\varepsilon}(\vartheta)=\inf _{q \in \Gamma_{\varepsilon}(\vartheta)} I(q)
$$

We will find for each $\varepsilon>0$ a candidate in $\Gamma_{\varepsilon}(\vartheta)$ and then send $\varepsilon$ to zero to obtain the minimizer, which is actually a heteroclinic solution.

For any $\varepsilon \in(0, \gamma), v>0$ define

$$
\sigma_{\varepsilon, v}=\inf _{\substack{z \notin B_{\varepsilon}(\mathscr{\mathscr { V }}),|z| \leqslant v, t \in \mathbb{R}}} V(t, z),
$$

then $\sigma_{\varepsilon, v}>0$ by our assumptions. For $v=+\infty$, we take $\sigma_{\varepsilon,+\infty}=\sigma_{\varepsilon}$ which is defined in $\left(A_{3}\right)$.

From now on, we always assume that

$$
0<\varepsilon<\min \left\{\varepsilon^{*}, 1\right\} .
$$


Lemma 3. Let $q \in E$. Then for any $v>0, r<s \in \mathbb{R}$ such that $q(t) \notin B_{\varepsilon}(\mathscr{V})$ and $|q(t)| \leqslant v$ for any $t \in[r, s]$,

$$
I(q) \geqslant \sqrt{2 \sigma_{\varepsilon, v}}|q(r)-q(s)|
$$

In particular, if $\sigma_{\varepsilon}>0$, then for any $r<s \in \mathbb{R}$ such that $q(t) \notin B_{\varepsilon}(\mathscr{V})$ for any $t \in[r, s]$,

$$
I(q) \geqslant \sqrt{2 \sigma_{\varepsilon}}|q(r)-q(s)| .
$$

Proof. Denote $l=|q(r)-q(s)|$ and $\tau=|r-s|$. Then

$$
l=\left|\int_{r}^{s} q^{\prime}(t) d t\right| \leqslant \int_{r}^{s}\left|q^{\prime}(t)\right| d t \leqslant \tau^{1 / 2}\left(\int_{r}^{s}\left|q^{\prime}(t)\right|^{2} d t\right)^{1 / 2} .
$$

Since $V \geqslant 0$, by assumptions, we have

$$
\begin{aligned}
I(q) & \geqslant \int_{r}^{s}\left\{\frac{1}{2}\left(q^{\prime}(t)\right)^{2}+V(t, q(t))\right\} d t \\
& \geqslant \frac{l^{2}}{2 \tau}+\sigma_{\varepsilon, v} \tau \geqslant \sqrt{2 \sigma_{\varepsilon, v}} l .
\end{aligned}
$$

Proof for the second part is similar.

Remark 4. The proof of Lemma 3 indicates that any function $q \in E$ with $I(q)<+\infty$ cannot oscillate too often between different points in $\mathscr{V}$ when the time approaches infinity; precisely, we will show $q( \pm \infty)$ exist and have limits in $\mathscr{V}$.

Lemma 5. Let $q \in E . I(q)<+\infty$ implies $q \in L^{\infty}(\mathbb{R}, \mathbb{R})$.

Proof. Suppose to the contrary that $q \notin L^{\infty}(\mathbb{R})$. Let $\varepsilon>0$ satisfy (15).

Case $1\left(\sigma_{\varepsilon}>0\right)$. If $\mathscr{V}$ is an infinite set, then we can find infinitely many nonoverlapping time intervals $\left\{\left(s_{i}, t_{i}\right)\right\}_{i \geqslant 1}$ such that $\left|q\left(s_{i}\right)-q\left(t_{i}\right)\right| \geqslant \gamma$ for $i \geqslant 1$ and $q(t) \notin B_{\varepsilon}(\mathscr{V})$ for $t \in \cup_{i \geqslant 1}\left(s_{i}, t_{i}\right)$, by Lemma 3 ,

$$
\begin{aligned}
I(q) & >\sum_{i=1}^{n} I_{s_{i}, t_{i}}(q) \\
& \geqslant \sqrt{2 \sigma_{\varepsilon}} \sum_{i=1}^{n}\left|q\left(s_{i}\right)-q\left(t_{i}\right)\right| \geqslant n \gamma \sqrt{2 \sigma_{\varepsilon}},
\end{aligned}
$$

for any $n \geqslant 1$, a contradiction.

If $\mathscr{V}$ is a finite set, let $z_{v}=\max _{z \in \mathscr{V}}|z|$, then $z_{v} \in(0, \infty)$. Since $q \notin L^{\infty}(\mathbb{R})$, there is a sequence of nonoverlapping time intervals, also denoted by $\left\{\left(s_{i}, t_{i}\right)\right\}_{i \geqslant 1}$, such that $\left|q\left(s_{i}\right)-q\left(t_{i}\right)\right| \geqslant$ 1 for $i \geqslant 1$ and $|q(t)|>\max \left\{|q(0)|, z_{v}+1\right\}$ for $t \in \cup_{i \geqslant 1}\left(s_{i}, t_{i}\right)$. Again Lemma 3 shows

$$
I(q) \geqslant n \sqrt{2 \sigma_{\varepsilon}}
$$

for any $n \geqslant 1$, also a contradiction.

Case $2\left(\liminf _{|z| \rightarrow \infty}|z| V(t, z)>0\right.$ uniformly in $\left.t \in \mathbb{R}\right)$. There are $z_{0}>0$ and $c_{1}>0$ such that $V(t, z) \geqslant c_{1}|z|^{-1}$ for $|z|>z_{0}$ and $t \in \mathbb{R}$. Let $n_{q}=\max \left\{[|q(0)|]+1,\left[z_{0}\right]+1\right\}([x]$ denotes the integral part of $x)$. Since $q \notin L^{\infty}(\mathbb{R})$, there is a sequence of nonoverlapping time intervals $\left\{\left(s_{j}, t_{j}\right)\right\}_{j \geqslant n_{q}}$ with $\left|q\left(s_{j}\right)\right|=j$, $\left|q\left(t_{j}\right)\right|=j+1$ and $|q(t)| \in[j, j+1]$ on $\left(s_{j}, t_{j}\right)$ this is possible via the continuity of $q(t)$. We claim that $\inf _{j \geqslant n_{q}}\left|t_{j}-s_{j}\right|>0$. Indeed, for $j \geqslant n_{q}$,

$$
\begin{aligned}
1 & =|| q\left(t_{j}\right)|-| q\left(s_{j}\right)|| \\
& \leqslant\left|q\left(t_{j}\right)-q\left(s_{j}\right)\right| \\
& \leqslant \int_{s_{j}}^{t_{j}}\left|q^{\prime}(t)\right| d t \\
& \leqslant\left|t_{j}-s_{j}\right|^{1 / 2}\left(\int_{s_{j}}^{t_{j}}\left|q^{\prime}(t)\right|^{2} d t\right)^{1 / 2},
\end{aligned}
$$

so

$$
\begin{aligned}
1 & \leqslant\left|t_{j}-s_{j}\right| \int_{r}^{s}\left|q^{\prime}(t)\right|^{2} d t \\
& \leqslant 2\left|t_{j}-s_{j}\right| I(q), \quad \forall j \geqslant n_{q},
\end{aligned}
$$

which implies

$$
\inf _{j \geqslant n_{q}}\left|t_{j}-s_{j}\right|>0
$$

Hence,

$$
\begin{aligned}
I(q) & \geqslant \sum_{j=n_{q}}^{n} \int_{s_{j}}^{t_{j}} V(t, q(t)) d t \\
& \geqslant c_{1} \sum_{j=n_{q}}^{n} \int_{s_{j}}^{t_{j}} \frac{1}{|q(t)|} d t \geqslant c_{1}\left(\inf _{j \geqslant n_{q}}\left|t_{j}-s_{j}\right|\right) \sum_{j=n_{q}}^{n} \frac{1}{j+1},
\end{aligned}
$$

for any $n \geqslant n_{q}$, a contradiction.

Therefore, in either case, we must have $q \in L^{\infty}(\mathbb{R})$.

The preceding lemma has an important corollary which will be used later; we prove it here.

Lemma 6. For $M>0$ and $u \in \mathscr{V}$, define

$$
Q_{u, M}=\{q \in E \mid q(-\infty)=u, I(q) \leqslant M\} .
$$

Then $Q_{u, M}$ is a bounded set in $C(\mathbb{R})$ with the usual uniform norm.

Proof. Without loss of generality, assume $u=0$. Let $\varepsilon>0$ satisfy (15). Suppose to the contrary that, for any $n \in \mathbb{N}$, there is an orbit $q_{n}$ such that $q_{n}(-\infty)=0, I\left(q_{n}\right) \leqslant M$ and $\sup _{t \in \mathbb{R}}\left|q_{n}(t)\right|>n$. Since $q_{n}(t)$ is continuous, there is $t_{n}^{*} \in \mathbb{R}$ for which $\left|q_{n}\left(t_{n}^{*}\right)\right|=n$ and $\left|q_{n}(t)\right| \leqslant n$ for $t \leqslant t_{n}^{*}$; this can be accomplished by taking as $t_{n}^{*}$ the first hitting time of $\left|q_{n}(t)\right|$ on the level $n$.

Case $1\left(\sigma_{\varepsilon}>0\right)$. If $\mathscr{V}$ is an infinite set, then for each $n \geqslant$ $[3 \gamma]+1, n \in \mathbb{N}$, there are $l_{n}$ nonoverlapping time intervals 
$\left\{\left(s_{n, i}, t_{n, i}\right)\right\}_{i \leqslant l_{n}}$ such that $\left|q_{n}\left(s_{n, i}\right)-q_{n}\left(t_{n, i}\right)\right| \geqslant \gamma$ for $1 \leqslant i \leqslant l_{n}$, $q_{n}(t) \notin B_{\varepsilon}(\mathscr{V})$ for $t \in \cup_{i=1}^{l_{n}}\left(s_{n, i}, t_{n, i}\right)$; moreover, the number of intervals $l_{n} \rightarrow \infty$ as $n \rightarrow \infty$. Then by Lemma 3 ,

$$
\begin{aligned}
I\left(q_{n}\right) & >\sum_{i=1}^{l_{n}} I_{s_{n, i}, t_{n, i}}\left(q_{n}\right) \geqslant \sqrt{2 \sigma_{\varepsilon}} \sum_{i=1}^{l_{n}}\left|q_{n}\left(s_{n, i}\right)-q_{n}\left(t_{n, i}\right)\right| \\
& \geqslant l_{n} \gamma \sqrt{2 \sigma_{\varepsilon}},
\end{aligned}
$$

for any $n \geqslant[3 \gamma]+1$, a contradiction.

If $\mathscr{V}$ is a finite set, let $z_{v}=\max _{z \in \mathscr{V}}|z|$, then $z_{v} \in(0, \infty)$. For each $n \geqslant\left[z_{v}\right]+1, n \in \mathbb{N}$, there is an $s_{n}<t_{n}^{*}$ with $\left|q_{n}\left(s_{n}\right)\right|=$ $z_{v}+1$ and $\left|q_{n}(t)\right| z_{v}+1$ for $t \in\left(s_{n}, t_{n}^{*}\right)$. Then Lemma 3 indicates

$$
I\left(q_{n}\right) \geqslant\left(n-\left(z_{v}+1\right)\right) \sqrt{2 \sigma_{\varepsilon}}
$$

for any $n \geqslant\left[z_{v}\right]+1$, also a contradiction.

Case $2\left(\liminf _{|z| \rightarrow \infty}|z| V(t, z)>0\right.$ uniformly in $\left.t \in \mathbb{R}\right)$. There are $z_{0}>0$ and $c_{1}>0$ such that $V(t, z) \geqslant c_{1}|z|^{-1}$ for $|z|>z_{0}$ and $t \in \mathbb{R}$. For each $n \geqslant 2$, there are $n-1$ nonoverlapping time intervals $\left\{\left(s_{n, i}, t_{n, i}\right)\right\}_{2 \leqslant i \leqslant n}$ such that $\left|q_{n}\left(s_{n, i}\right)\right|=i-1,\left|q_{n}\left(t_{n, i}\right)\right|=i$ and $\left|q_{n}(t)\right| \in[i-1, i]$ on $\left(s_{n, i}, t_{n, i}\right)$. The same argument that we used to obtain (24) shows

$$
\inf _{n \geqslant 2} \inf _{n \geqslant j \geqslant 2}\left|t_{n, j}-s_{n, j}\right|>0 .
$$

Hence

$$
\begin{aligned}
I\left(q_{n}\right) & \geqslant \sum_{j=\left[z_{0}\right]+2}^{n} \int_{s_{n, j}}^{t_{n, j}} V\left(t, q_{j}(t)\right) d t \\
& \geqslant c_{1} \sum_{j=\left[z_{0}\right]+2}^{n} \int_{s_{n, j}}^{t_{n, j}} \frac{1}{\left|q_{j}(t)\right|} d t \\
& \geqslant c_{1}\left(\inf _{n \geqslant 2} \inf _{n \geqslant j \geqslant 2}\left|t_{n, j}-s_{n, j}\right|\right) \sum_{j=\left[z_{0}\right]+2}^{n} \frac{1}{j},
\end{aligned}
$$

for any $n \geqslant\left[z_{0}\right]+2, n \in \mathbb{N}$, a contradiction.

Therefore, a constant $C_{0}>0$ depending only on $M$ and $u$ exists, for which $\sup _{t \in \mathbb{R}}|q(t)| \leqslant C_{0}$ for all $q \in Q_{u, M}$.

Lemma 7. Let $q \in E$. If $I(q)<+\infty$, then there are $\alpha, \beta \in \mathscr{V}$ such that $\lim _{t \rightarrow-\infty} q(t)=\alpha$ and $\lim _{t \rightarrow+\infty} q(t)=\beta$.

Proof. By Lemma 5, $q \in L^{\infty}(\mathbb{R}, \mathbb{R})$. Since $q \in C(\mathbb{R})$, then $\sup _{t \in \mathbb{R}}|q(t)| \leqslant Q$ for some $Q>0$. Therefore, the set

$$
\Omega(q,-\infty)=\left\{x: q\left(s_{k}\right) \longrightarrow x \text { for some } s_{k} \longrightarrow-\infty\right\}
$$

of accumulation points of $q$ at $-\infty$ is not empty. Moreover, $\Omega(q,-\infty) \cap \mathscr{V} \neq \emptyset$. Indeed, suppose there are $\delta \in(0, \gamma)$ and $r_{0}>0$ such that $q(t) \notin B_{\delta}(\mathscr{V})$ for all $t \leqslant-r_{0}$. Then, by definition of $\sigma_{\delta, \mathrm{Q}}$ in (14) with $\varepsilon=\delta$ and $v=Q$,

$$
I(q)>\int_{-\infty}^{-r_{0}}\{V(t, q)\} d t>\int_{-\infty}^{-r_{0}} \sigma_{\delta, Q} d t
$$

which shows $I(q)=+\infty$, a contradiction. Hence, $\Omega(q,-\infty) \cap$ $\mathscr{V}$ is not empty and there exists an $\alpha \in \Omega(q,-\infty) \cap \mathscr{V}$. Finally we claim $q(-\infty)=\alpha$. Suppose otherwise, there must be some $\delta_{1} \in\left(0, \varepsilon^{*}\right)$ such that $q(t)$ intersects $\partial B_{\delta_{1}}(\alpha)$ and $\partial B_{2 \delta_{1}}(\alpha)$ infinitely many times, then Lemma 3 implies that the energy along the orbit $q(t)$ would tend to infinity, contradicting the hypothesis. Thus, we have shown $q(t)$ tends to $\alpha$ as $t \rightarrow-\infty$. The other limit can be proved similarly.

Theorem 8. For any $\varepsilon \in\left(0, \varepsilon^{*}\right], \vartheta \in \mathscr{V} \backslash\{0\}$. There exists $q_{\varepsilon, \vartheta} \in \Gamma_{\varepsilon}(\vartheta)$ such that $q_{\varepsilon, \vartheta}$ minimizes the functional $I(q)$ on the set $\Gamma_{\mathcal{\varepsilon}}(\vartheta)$, that is, $I\left(q_{\varepsilon, \vartheta}\right)=c_{\varepsilon}(\vartheta)$.

Proof. Let $\left\{q_{\varepsilon, \vartheta, n}\right\} \in \Gamma_{\varepsilon}(\vartheta)$ be a minimizing sequence for (13),

$$
I\left(q_{\varepsilon, \vartheta, n}\right) \longrightarrow c_{\varepsilon}(\vartheta)=\inf _{u \in \Gamma_{\varepsilon}(\vartheta)} I(u) .
$$

For notational convenience, we will suppress subscripts $\varepsilon, \vartheta$ in what follows and simply write $q_{\varepsilon, \vartheta, n}=q_{n}$. Now since $\left\{q_{n}\right\}$ is a minimizing sequence, we have $I\left(q_{n}\right) \leqslant M$ for some $M>0$. Moreover, Lemma 6 shows that $\left\{q_{n}(0)\right\}$ is a bounded sequence; therefore, $\left\{q_{n}\right\}$ is bounded in $E$. Going if necessary to a subsequence, we may suppose that $\left\{q_{n}\right\}$ converges weakly in $E$ to an element $q \in E$. Sobolev imbedding theorem shows $q_{n} \rightarrow q$ in $C_{\text {loc }}^{1}(\mathbb{R})$. To show $q$ is the minimizer we are looking for, we need firstly to prove

$$
I(q)<+\infty \text {. }
$$

Indeed, for any $r<s \in \mathbb{R}$,

$$
I_{r, s}\left(q_{n}\right) \leqslant I\left(q_{n}\right) \leqslant M
$$

by the weakly lower semicontinuity of $I_{r, s}(\cdot)$,

$$
I_{r, s}(q)=\liminf _{n \rightarrow+\infty} I_{r, s}\left(q_{n}\right) \leqslant \liminf _{n \rightarrow+\infty} I\left(q_{n}\right)=\inf _{u \in \Gamma_{\varepsilon}(9)} I(u) \leqslant M .
$$

Clearly, the constant $M$ is independent of $r$ and $s$. Since $q \in E$, the above inequality implies $V(\cdot, q(\cdot)) \in L^{1}(\mathbb{R}, \mathbb{R})$, and

$$
I(q) \leqslant \inf _{u \in \Gamma_{\varepsilon}(\vartheta)} I(u) \leqslant M .
$$

Hence, (34) holds.

Next we show

$$
\lim _{t \rightarrow-\infty} q(t)=0, \quad \lim _{t \rightarrow+\infty} q(t)=\vartheta .
$$

Inequality (34) and Lemma 7 imply that there are $\alpha, \beta \in \mathscr{V}$ verifying

$$
\lim _{t \rightarrow-\infty} q(t)=\alpha, \quad \lim _{t \rightarrow+\infty} q(t)=\beta .
$$

But for each $n,\left(q_{n}(t), q_{n}^{\prime}(t)\right) \notin B_{\varepsilon}(\widetilde{\mathscr{V}} \backslash\{\widetilde{0}, \widetilde{\vartheta}\})$ for all $t \in \mathbb{R}$ and $q_{n} \rightarrow q$ in $C_{\text {loc }}^{1}(\mathbb{R})$, so $\left(q(t), q^{\prime}(t)\right) \notin B_{\varepsilon}(\widetilde{\mathscr{V}} \backslash\{\widetilde{0}, \widetilde{\mathcal{\vartheta}}\})$ for all $t \in$ $\mathbb{R}$. Therefore, $\alpha, \beta \in\{0, \vartheta\}$. For each $t \in \mathbb{R}$, there is $n(t) \in \mathbb{R}$ such that

$$
\left|q_{n}(t)-q(t)\right|<\varepsilon, \quad \forall n \geqslant n(t) .
$$


We claim $\beta=\vartheta$. Suppose to the contrary that

$$
\lim _{t \rightarrow+\infty} q(t)=0 .
$$

Then, there is $t_{0}>0$ such that

$$
|q(t)|<\varepsilon, \quad \forall t \geqslant t_{0} .
$$

Hence,

$$
\left|q_{n}(t)\right| \leqslant\left|q_{n}(t)-q(t)\right|+|q(t)|<2 \varepsilon,
$$

whenever $t \geqslant t_{0}$ and $n \geqslant n(t)$. In particular

$$
\left|q_{n(t)}(t)\right| \leqslant 2 \varepsilon, \quad \forall t \geqslant t_{0} .
$$

Let

$$
\begin{aligned}
& s_{n}=s_{n}(\varepsilon)=\sup \left\{t \in \mathbb{R} \mid q_{n}(t) \in \partial B_{2 \varepsilon}(0)\right\}, \\
& \bar{s}_{n}=\bar{s}_{n}(\varepsilon)=\inf \left\{t>s_{n} \mid q_{n}(t) \in \partial B_{4 \varepsilon}(0)\right\} .
\end{aligned}
$$

Since $q_{n} \in \Gamma_{\varepsilon}(\vartheta), s_{n}<\bar{s}_{n}$ are well defined for each $n$. The same argument that we used to obtain (24) shows

$$
\inf _{n}\left|\bar{s}_{n}-s_{n}\right| \geqslant c_{0},
$$

for some constant $c_{0}>0$. Whence, for $t \geqslant t_{0}$, by the definition of $s_{n}$ and $c_{0},\left|q_{n(t)}\left(\tau_{n(t)}\right)\right| \in(2 \varepsilon, 4 \varepsilon)$, and the mean value theorem,

$$
I\left(q_{n(t)}\right) \geqslant \int_{s_{n(t)}}^{s_{n(t)}+c_{0}} V\left(s, q_{n(t)}\right) d s=c_{0} V\left(\tau_{n(t)}, q_{n(t)}\left(\tau_{n(t)}\right)\right),
$$

where $\tau_{n(t)} \in\left(s_{n(t)}, s_{n(t)}+c_{0}\right)$. Inequality (44) also implies $t \leqslant$ $s_{n(t)}<\tau_{n(t)}$. Hence, by $\left(A_{4}\right), V\left(\tau_{n(t)}, q_{n(t)}\left(\tau_{n(t)}\right)\right) \rightarrow+\infty$ as $t \rightarrow+\infty$, which leads to a contradiction that $I\left(q_{n(t)}\right) \rightarrow+\infty$ as $t \rightarrow+\infty$. Similar argument shows that $\lim _{t \rightarrow-\infty} q(t)=0$ and the proof is completed.

Denote

$$
\begin{aligned}
\mathscr{G} & =\mathscr{G}(\varepsilon, \vartheta) \\
& =\left\{t \in \mathbb{R} \mid\left(q_{\varepsilon, \vartheta}(t), q_{\varepsilon, \vartheta}^{\prime}(t)\right) \in \partial B_{\varepsilon}(\widetilde{\mathscr{V}} \backslash\{\widetilde{0}, \widetilde{\vartheta}\})\right\} .
\end{aligned}
$$

Theorem 9. The minimizer $q=q_{\varepsilon, \vartheta}$ obtained in Theorem 8 is a classical solution on $\mathbb{R} \backslash \mathscr{G}$.

Proof. For any $t \in \mathbb{R} \backslash \mathscr{G}$, since $\mathbb{R} \backslash \mathscr{G}$ is an open set, there is a maximal open interval $\mathfrak{S} \subset \mathbb{R} \backslash \mathscr{G}$ containing $t$. Let $\varphi \in$ $C_{0}^{\infty}(\Im)$, then for $\delta>0$ sufficiently small $q+\delta \varphi \in \Gamma_{\mathcal{\varepsilon}}(\vartheta)$. Since $q$ minimize $I(\cdot)$ on $\Gamma_{\varepsilon}(\vartheta)$, then, for all $\varphi \in C_{0}^{\infty}(\mathfrak{I})$,

$$
\left(I^{\prime}(q), \varphi\right)=\int_{-\infty}^{\infty}\left\{q^{\prime \prime} \varphi^{\prime \prime}+q^{\prime} \varphi^{\prime}+V_{q}(t, q) \varphi\right\} d t=0 .
$$

Fix any $r<s \in \mathfrak{I}$, this equality also holds for any $\varphi \in$ $W_{0}^{2,2}([r, s])$. Hence, $q$ is a weak solution of the following system:

$$
\begin{gathered}
u^{\prime \prime \prime \prime}(t)-u^{\prime \prime}(t)+V_{q}(t, q)=0, \quad r<t<s ; \\
u(r)=q(r), \quad u(s)=q(s) ; \\
u^{\prime}(r)=q^{\prime}(r), \quad u^{\prime}(s)=q^{\prime}(s) ;
\end{gathered}
$$

the system has a unique $C^{4}$ solution, denoted by $v$. Then

$$
\int_{r}^{s}\left\{v^{\prime \prime} \varphi^{\prime \prime}+v^{\prime} \varphi^{\prime}+V_{q}(t, q) \varphi\right\} d t=0 .
$$

Combining (49) and (51) yields

$$
\int_{r}^{s}\left\{\left(q^{\prime \prime}-v^{\prime \prime}\right) \varphi^{\prime \prime}+\left(q^{\prime}-v^{\prime}\right) \varphi^{\prime}\right\} d t=0,
$$

for all $\varphi \in W_{0}^{2,2}([r, s])$. Since $q-v$ belongs to $E$, it follows that $q-v$ is constant. Noting the boundary conditions that $q$ coincides with $v$ at $r$ and $s$, we have $q=v \in C^{4}([r, s])$. Since $r, s \in \mathfrak{I}$ are arbitrary, it follows that $q \in C^{4}(\mathfrak{I})$, which completes the proof.

Define

$$
c_{\varepsilon}=\inf _{\vartheta \in \mathscr{\mathscr { V }} \backslash\{0\}} c_{\mathcal{\varepsilon}}(\vartheta) .
$$

Since $c_{\varepsilon}$ is nonincreasing as a function of $\varepsilon>0$, inf ${ }_{\varepsilon>0} c_{\varepsilon}$ exists and we may choose a decreasing sequence $\varepsilon_{i} \rightarrow 0$ such that

$$
c_{\varepsilon_{i}} \longrightarrow \inf _{\varepsilon>0} c_{\varepsilon} \text { as } i \longrightarrow \infty .
$$

By Lemma 6, for each $i$, the set of candidates for the infimum $c_{\varepsilon_{i}}$ is finite; hence, it is achieved by an element $\kappa_{i} \in \mathscr{V} \backslash\{0\}$, namely,

$$
c_{\varepsilon_{i}}=c_{\varepsilon_{i}}\left(\kappa_{i}\right)=\inf _{q \in \Gamma_{\varepsilon_{i}}\left(\kappa_{i}\right)} I(q) \text {. }
$$

Theorem 8 shows, for each $i$, there is a $q_{i} \in \Gamma_{\varepsilon_{i}}\left(\kappa_{i}\right)$ such that

$$
I\left(q_{i}\right)=\inf _{q \in \Gamma_{\varepsilon_{i}}\left(\kappa_{i}\right)} I(q) .
$$

Again by Lemma 6, $\left\{\kappa_{i}, i \geqslant 1\right\} \subset \mathscr{V} \backslash\{0\}$ must be a finite set and thus contains a constant subsequence $\left\{\kappa_{i_{k}}, k \geqslant 1\right\}$; in other words, there is a $\kappa \in\left\{\kappa_{i}, i \geqslant 1\right\}$ for which $\kappa_{i_{k}}=\kappa$, for all $k \geqslant 1$. Without loss of generality, we also denote this constant sequence by $\left\{\kappa_{i}, i \geqslant 1\right\}$ (correspondingly $\left\{\varepsilon_{i}, i \geqslant 1\right\}$ ), then

$$
c_{\varepsilon_{i}}=\inf _{q \in \Gamma_{\varepsilon_{i}}(\kappa)} I(q)=I\left(q_{i}\right)
$$

where $q_{i} \in \Gamma_{\varepsilon_{i}}(\kappa)$.

Theorem 10. For $i$ sufficiently large, $q_{i}$ is a heteroclinic solution connecting 0 and $\kappa$.

Proof. To finish the proof, we show that $q_{i}$ never touches $\partial B_{\varepsilon_{i}}(\widetilde{\mathscr{V}} \backslash\{\tilde{0}, \widetilde{\kappa}\})$ for $i$ large enough. Assume not, then there are $\left\{\chi_{j}\right\} \subset \mathscr{V} \backslash\{0, \kappa\}$ and $\left\{\tau_{j}\right\} \in \mathbb{R}$ such that $\left(q_{j}\left(\tau_{j}\right), q_{j}^{\prime}\left(\tau_{j}\right)\right) \in$ $\partial B_{\varepsilon_{j}}\left(\tilde{\chi}_{j}\right)$ and $\left(q_{j}(t), q_{j}^{\prime}(t)\right) \notin \partial B_{\varepsilon_{j}}\left(\tilde{\chi}_{j}\right)$ for $t<\tau_{j}$; precisely, $\tau_{j}$ is the first hitting time of $q_{j}$ on $\partial B_{\varepsilon_{j}}\left(\tilde{\chi}_{j}\right)$. An application of Lemma 6 shows that $\left\{\chi_{j}\right\}$ is bounded, so it must contain a constant subsequence which we still denote by $\left\{\chi_{j}\right\}$, that is, $\chi_{j} \equiv \chi \in \mathscr{V} \backslash\{0, \kappa\}$ for $j \geqslant 1$. Now, two situations may occur, for infinitely many $j$, (i) $\left(q_{j}, q_{j}^{\prime}\right)$ reaches $\partial B_{\varepsilon_{j}}(\widetilde{\mathscr{V}} \backslash\{\tilde{\chi}\})$ before $\partial B_{\varepsilon_{j}}(\widetilde{\mathscr{V}} \backslash\{\widetilde{\kappa}\})$ or (ii) touches $\partial B_{\varepsilon_{j}}(\widetilde{\mathscr{V}} \backslash\{\widetilde{\kappa}\})$ before $\partial B_{\varepsilon_{j}}(\widetilde{\mathscr{V}} \backslash\{\tilde{\chi}\})$. 
Since $q_{j} \in \Gamma_{\varepsilon_{j}}(\kappa), \varepsilon_{j}$ is a decreasing sequence of $j$ and $c_{\varepsilon_{j}}$ is nonincreasing in $j$; hence,

$$
I\left(q_{j}\right)=c_{\varepsilon_{j}} \leqslant c_{\varepsilon_{1}}<+\infty,
$$

which by Lemma 6 implies that $\left\{q_{j}\right\}$ must be uniformly bounded on $\mathbb{R}$, say $\sup _{s \in \mathbb{R}}\left|q_{j}(s)\right|<M$ for all $j$.

Case $1\left(\left(q_{j}(t), q_{j}^{\prime}(t)\right) \notin \bar{B}_{\varepsilon_{j}}(\widetilde{\kappa})\right.$ for all $\left.t<\tau_{j}\right)$. Before stepping forward, we need to show $\left\{\tau_{j}\right\}$ is bounded both from above and below. Suppose $\left\{\tau_{j}\right\}$ is unbounded from above. Let

$$
\begin{aligned}
& s_{j}=\sup \left\{t \in \mathbb{R} \mid\left(q_{j}(t), q_{j}^{\prime}(t)\right) \in \partial B_{\gamma}(\tilde{\chi})\right\}, \\
& \bar{s}_{j}=\inf \left\{t>s_{j} \mid\left(q_{j}(t), q_{j}^{\prime}(t)\right) \in \partial B_{\gamma}(\widetilde{\kappa})\right\} .
\end{aligned}
$$

For $j$ large enough, $\gamma>\varepsilon_{j}$, so $s_{j}>\tau_{j}$ by definition of $\tau_{j}$. Hence, $\left\{s_{j}\right\}$ is unbounded from above. Without loss of generality; we may suppose $\lim _{j \rightarrow \infty} s_{j}=+\infty$. Similar to (46) it can be shown that

$$
\inf _{j}\left|s_{j}-\bar{s}_{j}\right|>c_{1}
$$

for some positive constant $c_{1}>0$. Hence,

$$
\begin{aligned}
+\infty & >c_{\varepsilon_{j}}=I\left(q_{j}\right) \\
& \geqslant \int_{s_{j}}^{\bar{s}_{j}} V\left(s, q_{j}(s)\right) d s \\
& =\left(\bar{s}_{j}-s_{j}\right) V\left(\widehat{s}_{j}, q_{j}\left(\widehat{s}_{j}\right)\right) \\
& >c_{1} V\left(\widehat{s}_{j}, q_{j}\left(\widehat{s}_{j}\right)\right),
\end{aligned}
$$

where $s_{j}<\widehat{s}_{j}<\bar{s}_{j}$. Now assumption $\left(A_{4}\right)$ and the uniform boundedness of $\left\{q_{j}\right\}$ indicate that $\left\{\widehat{s}_{j}\right\}$ is bounded, which is a contradiction. Thus, $\left\{\tau_{j}\right\}$ must have an upper bound. Similarly it can be proved that $\left\{\tau_{j}\right\}$ is also bounded from below by considering $r_{j}=\sup \left\{t \in \mathbb{R} \mid\left(q_{j}(t), q_{j}^{\prime}(t)\right) \in \partial B_{\gamma}(\widetilde{0})\right\}$ and $\bar{r}_{j}=\inf \left\{t>r_{j} \mid\left(q_{j}(t), q_{j}^{\prime}(t)\right) \in \partial B_{\gamma}(\widetilde{\kappa})\right\}$. Hence, $\left\{\tau_{j}\right\}$ is bounded.

Define

$$
\varsigma_{j}(t)= \begin{cases}\chi, & \text { for } t \geqslant \tau_{j}+\varepsilon_{j}^{1 / 3} ; \\ P_{j}\left(t-\tau_{j}\right), & \text { for } t \in\left[\tau_{j}, \tau_{j}+\varepsilon_{j}^{1 / 3}\right) ; \\ q_{j}(t), & \text { for } t<\tau_{j},\end{cases}
$$

where $P_{j}$ is a polynomial defined as

$$
\begin{gathered}
P_{j}(t)=a_{j}+b_{j} t+c_{j} t^{2}+d_{j} t^{3}, \\
a_{j}=q_{j}\left(\tau_{j}\right), \quad b_{j}=q_{j}^{\prime}\left(\tau_{j}\right), \\
c_{j}=-\varepsilon_{j}^{-2 / 3}\left[3\left(q_{j}\left(\tau_{j}\right)-\chi\right)+2 \varepsilon_{j}^{1 / 3} q_{j}^{\prime}\left(\tau_{j}\right)\right], \\
d_{j}=\varepsilon_{j}^{-1}\left[2\left(q_{j}\left(\tau_{j}\right)-\chi\right)+\varepsilon_{j}^{1 / 3} q_{j}^{\prime}\left(\tau_{j}\right)\right] .
\end{gathered}
$$

It is easy to verify the boundary conditions

$$
\begin{gathered}
\left(P_{j}(0), P_{j}^{\prime}(0)\right)=\left(q_{j}\left(\tau_{j}\right), q_{j}^{\prime}\left(\tau_{j}\right)\right), \\
\left(P_{j}\left(\varepsilon_{j}^{1 / 3}\right), P_{j}^{\prime}\left(\varepsilon_{j}^{1 / 3}\right)\right)=(\chi, 0) .
\end{gathered}
$$

In addition, $\lim _{j \rightarrow \infty} a_{j}=0, \lim _{j \rightarrow \infty} b_{j}=0, \lim _{j \rightarrow \infty} c_{j}=0$, and $\left\{d_{j}\right\}$ is bounded.

Comparing the energy of $q_{j}$ and $\varsigma_{j}$, we have

$$
\begin{aligned}
I\left(q_{j}\right)-I\left(\varsigma_{j}\right) & =I_{\tau_{j},+\infty}\left(q_{j}\right)-I_{\tau_{j},+\infty}\left(\varsigma_{j}\right) \\
& =I_{\tau_{j},+\infty}\left(q_{j}\right)-I_{\tau_{j}, \tau_{j}+\varepsilon_{j}^{1 / 3}}\left(\varsigma_{j}\right)-I_{\tau_{j}+\varepsilon_{j}^{1 / 3},+\infty}\left(\varsigma_{j}\right) \\
& \geqslant \gamma \sqrt{2 \sigma_{\gamma}}-I_{\tau_{j}, \tau_{j}+\varepsilon_{j}^{1 / 3}}\left(\varsigma_{j}\right)-I_{\tau_{j}+\varepsilon_{j}^{1 / 3},+\infty}\left(\varsigma_{j}\right) .
\end{aligned}
$$

By assumption $\left(A_{5}\right)$,

$$
I_{\tau_{j}+\varepsilon_{j}^{1 / 3},+\infty}\left(\varsigma_{j}\right)<\int_{-\infty}^{\infty} V(t, \chi) d t<\gamma \sqrt{\sigma_{\gamma}}
$$

Moreover, since $\left\{\varsigma_{j}\right\}$ is uniformly bounded on $\mathbb{R}$ and $\left\{\tau_{j}\right\}$ is bounded, it readily follows that

$$
\int_{\tau_{j}}^{\tau_{j}+\varepsilon_{j}^{1 / 3}} V\left(t, \varsigma_{j}\right) d t \longrightarrow 0 \quad \text { as } j \longrightarrow \infty .
$$

Meanwhile, by construction,

$$
\begin{gathered}
\int_{\tau_{j}}^{\tau_{j}+\varepsilon_{j}^{1 / 3}}\left(\varsigma_{j}^{\prime \prime}\right)^{2}=\int_{\tau_{j}}^{\tau_{j}+\varepsilon_{j}^{1 / 3}}\left(2 c_{j}+6 d_{j} t\right)^{2} d t \longrightarrow 0, \\
\int_{\tau_{j}}^{\tau_{j}+\varepsilon_{j}^{1 / 3}}\left(\varsigma_{j}^{\prime}\right)^{2}=\int_{\tau_{j}}^{\tau_{j}+\varepsilon_{j}^{1 / 3}}\left(b_{j}+2 c_{j} t+3 d_{j} t^{2}\right)^{2} d t \longrightarrow 0 .
\end{gathered}
$$

So

$$
\lim _{j \rightarrow+\infty} I_{\tau_{j}, \tau_{j}+\varepsilon_{j}^{1 / 3}}\left(\varsigma_{j}\right)=0
$$

Therefore, for $j$ large enough,

$$
\begin{aligned}
I\left(q_{j}\right)-I\left(\varsigma_{j}\right) & \geqslant \gamma \sqrt{2 \sigma_{\gamma}}-\frac{\sqrt{2}-1}{2} \gamma \sqrt{\sigma_{\gamma}}-\gamma \sqrt{\sigma_{\gamma}} \\
& >\frac{\sqrt{2}-1}{2} \gamma \sqrt{\sigma_{\gamma}},
\end{aligned}
$$

which is impossible.

Case $2\left(\left(q_{j}(t), q_{j}^{\prime}(t)\right) \in \partial B_{\varepsilon_{j}}(\{\widetilde{\kappa}\})\right.$ for some $\left.s_{j}<\tau_{j}\right)$. A contradiction can also be reached by similar arguments.

Either case leads to a contradiction; therefore, for $i$ sufficiently large, the orbit of $q_{i}=q_{\varepsilon_{i}, \kappa}$ must keep some distance away from $\widetilde{\mathscr{V}} \backslash\{\widetilde{0}, \widetilde{\kappa}\}$, and hence it is a classical solution, and the proof is complete. 
Proof of Theorem 1. Theorem 10 shows for every $z \in \mathscr{V}$, there is heteroclinic solution of (1) emanating from $z$. If we consider the $\operatorname{set} \bar{\Gamma}_{\varepsilon}(\vartheta) \subset E$ of function $q(t)$ for which $q(+\infty)=$ $0, q(-\infty)=\vartheta \in \mathscr{V} \backslash\{0\}$ and $\left(q(t), q^{\prime}(t)\right) \notin B_{\varepsilon}(\widetilde{\mathscr{V}} \backslash\{\widetilde{0}, \widetilde{\mathcal{\vartheta}}\})$, for all $t \in \mathbb{R}$; then the proof for $\Gamma_{\varepsilon}(\vartheta)$ works equally well for $\bar{\Gamma}_{\varepsilon}(\vartheta)$, and so, for every $z \in \mathscr{V}$, there is heteroclinic solution of (1) terminating at $z$.

\section{Heteroclinic Solution in Periodic Case}

In the last section, we consider the case where $V(t, z)$ is periodic in $t$ with period $T>0$. In this case, the assumptions and proof can be simplified. For completeness, we devote this section to the periodic case. Since most results in the last section can be carried out verbatim, we just present those that are different. We make the following assumptions:

$\left(H_{1}\right) V(t, q) \in C^{2}(\mathbb{R} \times \mathbb{R}), V \geqslant 0 ;$

$\left(H_{2}\right)$ the set $\mathscr{V}=\left\{z \in \mathbb{R} \mid\right.$ for all $t, V(t, z)=0, V_{q}(t, z)=$ 0 and $\left.V_{q q}(t, z) \geqslant 0\right\}$ is discrete and has at least two points, and

$$
\gamma \equiv \frac{1}{3} \inf \{|x-y| \mid x \neq y \in \mathscr{V}\}>0
$$

We also assume without no loss of generality $0 \in \mathscr{V}$.

$\left(H_{3}\right)$ There is a constant $V_{0}>0$ such that $\liminf _{|q| \rightarrow \infty}$ $V(t, q) \geqslant V_{0}$ uniformly for $t \in \mathbb{R}$.

$\left(H_{4}\right)$ For each $z \in \mathscr{V}$,

$$
\int_{-\infty}^{\infty} V(t, z) d t<\gamma \sigma_{\gamma}^{1 / 2}
$$

Note that $\left(A_{4}\right)$ is no longer needed in the periodic case. $\left(H_{3}\right)$ is indeed a special case of $\left(A_{3}\right)$, we are using $\left(H_{3}\right)$ instead of $\left(A_{3}\right)$ only for illustrative purpose. As before, we work on the space

$$
E=\left\{q \in W_{\mathrm{loc}}^{2,2}(\mathbb{R}, \mathbb{R}) \mid \int_{-\infty}^{\infty}\left(\left|q^{\prime \prime}\right|^{2}+\left|q^{\prime}\right|^{2}\right) d t<\infty\right\},
$$

with the norm

$$
\|q\|^{2}=\int_{-\infty}^{\infty}\left(\left|q^{\prime \prime}\right|^{2}+\left|q^{\prime}\right|^{2}\right) d t+|q(0)|^{2}
$$

We will use the shift operator $\sigma_{j}$ defined for each $j$ as

$$
\sigma_{j} q=q(t-j T)
$$

Clearly, if $q$ is a heteroclinic solution, so is $\tau_{j} q$ for all $j$. Recall $\Gamma_{\varepsilon}(\zeta), \zeta \in \mathscr{V} \backslash\{0\}$ is the set of $q \in E$ verifying

(i) $\lim _{t \rightarrow-\infty} q(t)=0, \lim _{t \rightarrow+\infty} q(t)=\zeta$;

(ii) $\left(q(t), q^{\prime}(t)\right) \notin B_{\varepsilon}(\widetilde{\mathscr{V}} /\{\widetilde{0}, \widetilde{\vartheta}\})$, for all $t \in \mathbb{R}$; where $\widetilde{\mathscr{V}}=$ $\left\{(z, 0) \in \mathbb{R}^{2} \mid z \in \mathscr{V}\right\}$.
$\Gamma_{\varepsilon}(\zeta)$ is not empty, we may consider the minimization problem

$$
c_{\varepsilon}(\zeta)=\inf _{q \in \Gamma_{\varepsilon}(\zeta)} I(q) .
$$

We shall follow the lines of argument in previous section, and similar proofs will be omitted. For example, Lemmas 3, 5,6 , and 7 remain valid in the setting of this section.

Theorem 11. For any $\varepsilon \in(0, \gamma], \widetilde{\zeta} \in \widetilde{\mathscr{V}} \backslash\{\widetilde{0}\}$. There exists $q_{\varepsilon, \zeta} \in \Gamma_{\varepsilon}(\zeta)$ such that $q_{\varepsilon, \zeta}$ minimizes the functional $I(q)$ on the set $\Gamma_{\varepsilon}(\zeta)$, that is, $I\left(q_{\varepsilon, \zeta}\right)=c_{\varepsilon}(\zeta)$.

Proof. Let $\left\{q_{n}\right\}$ be a minimizing sequence for (77). As in the preceding section, it is bounded in $E$ and we may choose a subsequence, also denoted by $\left\{q_{n}\right\}$, converging weakly in $E$ to some $q \in E$ and $q_{n} \rightarrow q$ in $C_{\text {loc }}^{1}(\mathbb{R})$. Moreover,

$$
I(q)<+\infty \text {. }
$$

It remains to show

$$
\lim _{t \rightarrow-\infty} q(t)=0, \quad \lim _{t \rightarrow+\infty} q(t)=\zeta .
$$

Inequality (78) and Lemma 7 imply that there are $\widetilde{\alpha}, \widetilde{\beta} \in \widetilde{\mathscr{V}}$ verifying

$$
\lim _{t \rightarrow-\infty} q(t)=\alpha, \quad \lim _{t \rightarrow+\infty} q(t)=\beta .
$$

Since $V(t, u)$ is periodic in $t$, if $\left\{q_{n}\right\}$ is the minimizing sequence, so is $\sigma_{j_{n}} q_{n}$ for any sequence $\left\{j_{n}\right\} \subset \mathbb{N}$ and $I\left(q_{n}\right)=$ $I\left(\sigma_{j_{n}} q_{n}\right)$. Whence, with $\left\{j_{n}\right\}$ being appropriately chosen and $\left\{q_{n}\right\}$ replaced with $\left\{\sigma_{j_{n}} q_{n}\right\}$, we are safe to suppose that $q_{n}(t) \in$ $B_{\varepsilon}(0)$ for $t \leqslant 0$ and $q_{n}(s) \in \partial B_{\varepsilon}(0)$ for some $s \in[0, T]$. Noting for each $n,\left(q_{n}(t), q_{n}^{\prime}(t)\right) \notin B_{\varepsilon}(\widetilde{\mathscr{V}} \backslash\{\widetilde{0}, \widetilde{\zeta}\})$ for all $t \in \mathbb{R}$ and $q_{n} \rightarrow q$ in $C_{\text {loc }}^{1}(\mathbb{R})$, we have $\left(q(t), q^{\prime}(t)\right) \notin B_{\varepsilon}(\widetilde{\mathscr{V}} \backslash\{\tilde{0}, \tilde{\zeta}\})$ for all $t \in \mathbb{R}$ and so $\widetilde{\alpha}, \widetilde{\beta} \in\{\widetilde{0}, \widetilde{\zeta}\}$. Since $q_{n}(t) \in B_{\varepsilon}(0)$ for $t \leqslant 0$, $\alpha \in\{0, \zeta\} \cap \bar{B}_{\varepsilon}(0)=\{0\}$, so $\alpha=0$.

It remains to show $\beta=\zeta$. Note $\beta=q(+\infty) \in\{0, \zeta\}$. Suppose that $q(+\infty)=0$. Choose $\delta>0$, for the time being we only assume that $4 \delta<\varepsilon$. Since $q^{\prime}(+\infty)=0$, there is a $t_{\delta}>T$ such that $q(t), q^{\prime}(t) \in B_{\delta}(0)$ for all $t>t_{\delta}$. Since $q_{n} \rightarrow$ $q$ in $C_{\text {loc }}^{1}(\mathbb{R})$, so there is $n_{\delta}$, which depends on $\delta$, such that $q_{n}\left(t_{\delta}\right), q_{n}^{\prime}\left(t_{\delta}\right) \in B_{2 \delta}(0)$ for $n$ larger than $n_{\delta}$. But $q_{n}(s) \in \partial B_{\varepsilon}(0)$ for some $s \in[0, T]$, so $q_{n}$ intersects $\partial B_{2 \delta}(0)$ and $\partial B_{\varepsilon}(0)$. By Lemma 3, we have

$$
I\left(q_{n}\right) \geqslant \frac{\varepsilon}{2} \sqrt{2 \sigma_{\varepsilon / 2}}+I_{t_{\delta},+\infty}\left(q_{n}\right) .
$$

Now construct a sequence as follows:

$$
\varsigma_{n}(t)= \begin{cases}0, & \text { for } t \leqslant t_{\delta}-t_{n} ; \\ P_{n}\left(t-t_{\delta}+t_{n}\right), & \text { for } t \in\left(t_{\delta}-t_{n}, t_{\delta}\right] ; \\ q_{n}(t), & \text { for } t>t_{\delta},\end{cases}
$$

where

$$
t_{n}= \begin{cases}\sqrt[3]{\left|q_{n}\left(t_{\delta}\right)\right|+\left|q_{n}^{\prime}\left(t_{\delta}\right)\right|}, & \text { if } \sqrt[3]{\left|q_{n}\left(t_{\delta}\right)\right|+\left|q_{n}^{\prime}\left(t_{\delta}\right)\right|} \neq 0, \\ 1, & \text { if } \sqrt[3]{\left|q_{n}\left(t_{\delta}\right)\right|+\left|q_{n}^{\prime}\left(t_{\delta}\right)\right|}=0\end{cases}
$$


and $P_{n}(t)=g_{n} t^{2}+h_{n} t^{3}$ is a polynomial with coefficients

$$
\begin{aligned}
& g_{n}=t_{n}^{-2}\left[3 q_{n}\left(t_{\delta}\right)-t_{n} q_{n}^{\prime}\left(t_{\delta}\right)\right], \\
& h_{n}=t_{n}^{-3}\left[t_{n} q_{n}^{\prime}\left(t_{\delta}\right)-2 q_{n}\left(t_{\delta}\right)\right] .
\end{aligned}
$$

It is easy to check that $P_{n}(t)$ satisfies

$$
\begin{gathered}
\left(P_{n}(0), P_{n}^{\prime}(0)\right)=(0,0), \\
\left(P_{n}\left(t_{n}\right), P_{n}^{\prime}\left(t_{n}\right)\right)=\left(q_{n}\left(t_{\delta}\right), q_{n}^{\prime}\left(t_{\delta}\right)\right) .
\end{gathered}
$$

Moreover, $q_{n}\left(t_{\delta}\right), q_{n}^{\prime}\left(t_{\delta}\right)$ tends to zero if $\delta \rightarrow 0$ and $n>n_{\delta}$. By construction $\varsigma_{n} \in \Gamma_{\varepsilon}(\zeta)$. So far, we only used the fact $0<$ $4 \delta<\varepsilon$, we can fix $\delta$ smaller still such that, for all $n>n_{\delta}$,

$$
\begin{gathered}
I_{t_{\delta}-t_{n}, t_{\delta}}\left(\varsigma_{n}\right) \\
=\int_{t_{\delta}-t_{n}}^{t_{\delta}}\left\{\frac{1}{2}\left(2 g_{n}+6 h_{n} t\right)^{2}+\frac{1}{2}\left(2 g_{n} t+3 h_{n} t^{2}\right)^{2}+V\left(t, \varsigma_{n}\right)\right\} d t, \\
I_{t_{\delta}-t_{n}, t_{\delta}}\left(\varsigma_{n}\right)<\frac{\varepsilon}{4} \sqrt{2 \sigma_{\varepsilon / 2}} .
\end{gathered}
$$

Then

$$
\begin{aligned}
I\left(q_{n}\right) & -I\left(\varsigma_{n}\right) \\
& =I_{-\infty, t_{\delta}}\left(q_{n}\right)-I_{t_{\delta}-t_{n}, t_{\delta}}\left(\varsigma_{n}\right) \\
& \geqslant \frac{\varepsilon}{2} \sqrt{2 \sigma_{\varepsilon / 2}}-I_{t_{\delta}-t_{n}, t_{\delta}}\left(\varsigma_{n}\right) \\
& >\frac{\varepsilon}{4} \sqrt{2 \sigma_{\varepsilon / 2}},
\end{aligned}
$$

for $n>n_{\delta}$. This implies

$$
\begin{gathered}
\liminf _{n \rightarrow+\infty} I\left(q_{n}\right)-\frac{\varepsilon}{4} \sqrt{2 \sigma_{\varepsilon / 2}} \\
\geqslant \liminf _{n \rightarrow+\infty} I\left(\varsigma_{n}\right) \\
\geqslant \inf _{q \in \Gamma_{\varepsilon}(\zeta)} I(q) \\
=\liminf _{n \rightarrow+\infty} I\left(q_{n}\right),
\end{gathered}
$$

which is impossible. The proof is complete.

Theorem 12. There is a pair of orbits $q_{0, \zeta}, q_{\zeta, 0}$ which connect 0 and $\zeta$, one emanating from 0 and the other terminating at 0 .

Proof. The proof is similar to Theorems 8 and 10 in [9].

Lastly, we observe the following.

Theorem 13. For the solution $q=q_{0, \zeta}$ or $q_{\zeta, 0}$ obtained above, $\lim _{n \rightarrow \pm \infty} q^{\prime \prime}(t)=0, \lim _{n \rightarrow \pm \infty} q^{\prime \prime \prime}(t)=0$.

Proof. Consider $q=q_{0, \zeta}$. By $\left(H_{2}\right)$, there are positive constants $r^{*}, c_{1}, c_{2}$ such that

$$
\begin{gathered}
V(t, u) c_{1} \geqslant|u-\zeta|^{2}, \\
\left|V_{z}(t, u)\right| \leqslant c_{2}|u-\zeta|,
\end{gathered}
$$

whenever $|u-\zeta| \leqslant r^{*}$. Since $q(+\infty)=\zeta,|q(t)-\zeta| \leqslant r^{*}$ for $t$ larger than some $t^{*}>0$.

From (89) and the Euler equation,

$$
q^{\prime \prime \prime \prime}-q^{\prime \prime}+V_{z}(t, q)=0,
$$

we have

$$
\begin{aligned}
\int_{t^{*}}^{+\infty} & \left|q^{\prime \prime \prime \prime}(t)\right|^{2} d t \\
& \leqslant 2 \int_{t^{*}}^{+\infty}\left|q^{\prime \prime}(t)\right|^{2} d t+2 \int_{t^{*}}^{+\infty}\left|V_{z}(t, q)\right|^{2} d t \\
& \leqslant 2 \int_{t^{*}}^{+\infty}\left|q^{\prime \prime}(t)\right|^{2} d t+2 c_{2}^{2} \int_{t^{*}}^{+\infty}|q(t)-\zeta|^{2} d t \\
& =2 \int_{t^{*}}^{+\infty}\left|q^{\prime \prime}(t)\right|^{2} d t+2 \frac{c_{2}^{2}}{c_{1}} \int_{t^{*}}^{+\infty} c_{1}|q(t)-\zeta|^{2} d t \\
& \leqslant 2 \int_{t^{*}}^{+\infty}\left|q^{\prime \prime}(t)\right|^{2} d t+2 \frac{c_{2}^{2}}{c_{1}} \int_{t^{*}}^{+\infty} V(t, q(t)) d t \\
& <\infty .
\end{aligned}
$$

An application of the interpolation inequality yields

$$
\begin{aligned}
& \int_{t^{*}}^{+\infty}\left|q^{\prime \prime \prime}(t)\right|^{2} d t \\
& \quad \leqslant K \int_{t^{*}}^{+\infty}\left|q^{\prime \prime}(t)\right|^{2} d t+K \int_{t^{*}}^{+\infty}\left|q^{\prime \prime \prime \prime}(t)\right|^{2} d t<\infty,
\end{aligned}
$$

where $K$ is a positive constant.

Therefore, we have proved that

$$
\lim _{n \rightarrow+\infty} q^{\prime \prime}(t)=\lim _{n \rightarrow+\infty} q^{\prime \prime \prime}(t)=0 .
$$

The limit for negative infinity can be derived similarly. The proof is complete.

\section{Acknowledgment}

This work was supported by NSFC under Grant no. 11126035 and partly NSFC Grant no. 11201016.

\section{References}

[1] L. A. Peletier and W. C. Troy, "Spatial patterns described by the extended Fisher-Kolmogorov (EFK) equation: kinks," Differential and Integral Equations, vol. 8, no. 6, pp. 1279-1304, 1995.

[2] L. A. Peletier and W. C. Troy, "A topological shooting method and the existence of kinks of the extended Fisher-Kolmogorov equation," Topological Methods in Nonlinear Analysis, vol. 6, no. 2, pp. 331-355, 1995.

[3] L. A. Peletier and W. C. Troy, "Chaotic spatial patterns described by the extended Fisher-Kolmogorov equation," Journal of Differential Equations, vol. 129, no. 2, pp. 458-508, 1996.

[4] L. A. Peletier and W. C. Troy, "Spatial patterns described by the extended Fisher-Kolmogorov equation: periodic solutions," SIAM Journal on Mathematical Analysis, vol. 28, no. 6, pp. 13171353, 1997. 
[5] W. D. Kalies and R. C. A. M. VanderVorst, "Multitransition homoclinic and heteroclinic solutions of the extended FisherKolmogorov equation," Journal of Differential Equations, vol. 131, no. 2, pp. 209-228, 1996.

[6] W. D. Kalies, J. Kwapisz, and R. C. A. M. VanderVorst, "Homotopy classes for stable connections between Hamiltonian saddle-focus equilibria," Communications in Mathematical Physics, vol. 193, no. 2, pp. 337-371, 1998.

[7] W. D. Kalies, J. Kwapisz, J. B. VandenBerg, and R. C. A. M. VanderVorst, "Homotopy classes for stable periodic and chaotic patterns in fourth-order Hamiltonian systems," Communications in Mathematical Physics, vol. 214, no. 3, pp. 573-592, 2000.

[8] Y. Ruan, "Periodic and homoclinic solutions of a class of fourth order equations," The Rocky Mountain Journal of Mathematics, vol. 41, no. 3, pp. 885-907, 2011.

[9] L. Yeun, "Heteroclinic solutions for fourth order equations of EFK type," Forthcoming.

[10] D. Bonheure and L. Sanchez, "Heteroclinic orbits for some classes of second and fourth order differential equations," in Handbook of Differential Equations: Ordinary Differential Equations, A. Canada, P. Drabek, and A. Fonda, Eds., vol. 3, chapter 2, pp. 103-202, Elsevier/North-Holland, Amsterdam, The Netherlands, 2006.

[11] P. H. Rabinowitz, "Periodic and heteroclinic orbits for a periodic Hamiltonian system," Annales de l'Institut Henri Poincaré, Analyse Non Linéaire, vol. 6, no. 5, pp. 331-346, 1989.

[12] P. H. Rabinowitz, "Homoclinic and heteroclinic orbits for a class of Hamiltonian systems," Calculus of Variations and Partial Differential Equations, vol. 1, no. 1, pp. 1-36, 1993.

[13] M. Izydorek and J. Janczewska, "Heteroclinic solutions for a class of the second order Hamiltonian systems," Journal of Differential Equations, vol. 238, no. 2, pp. 381-393, 2007.

[14] Y. Ruan, "Notes on a class of one-dimensional Landau-Brazovsky models," Archiv der Mathematik, vol. 93, no. 1, pp. 77-86, 2009. 


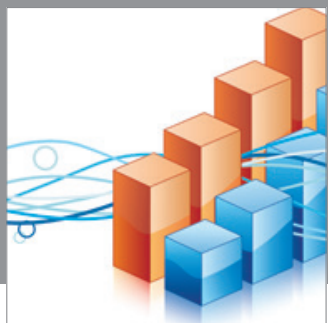

Advances in

Operations Research

mansans

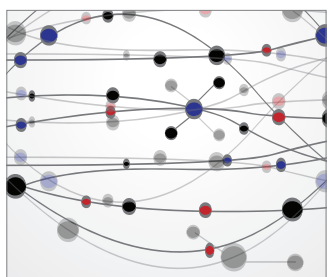

The Scientific World Journal
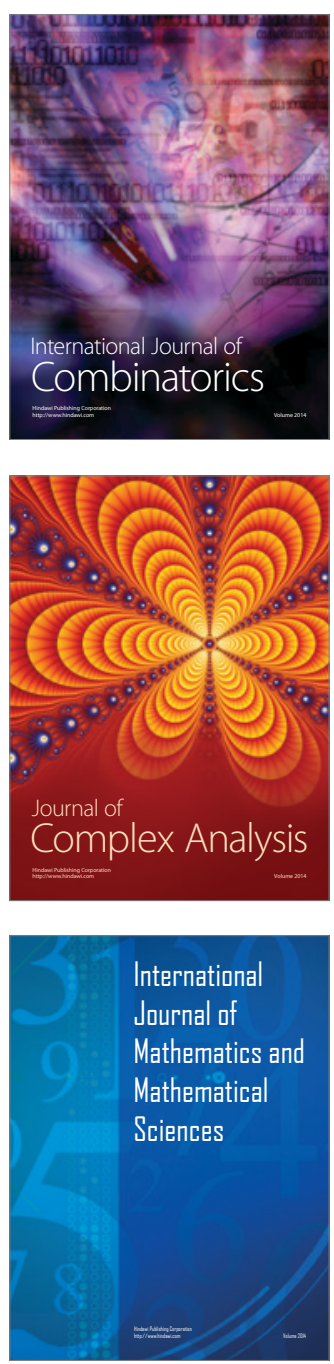
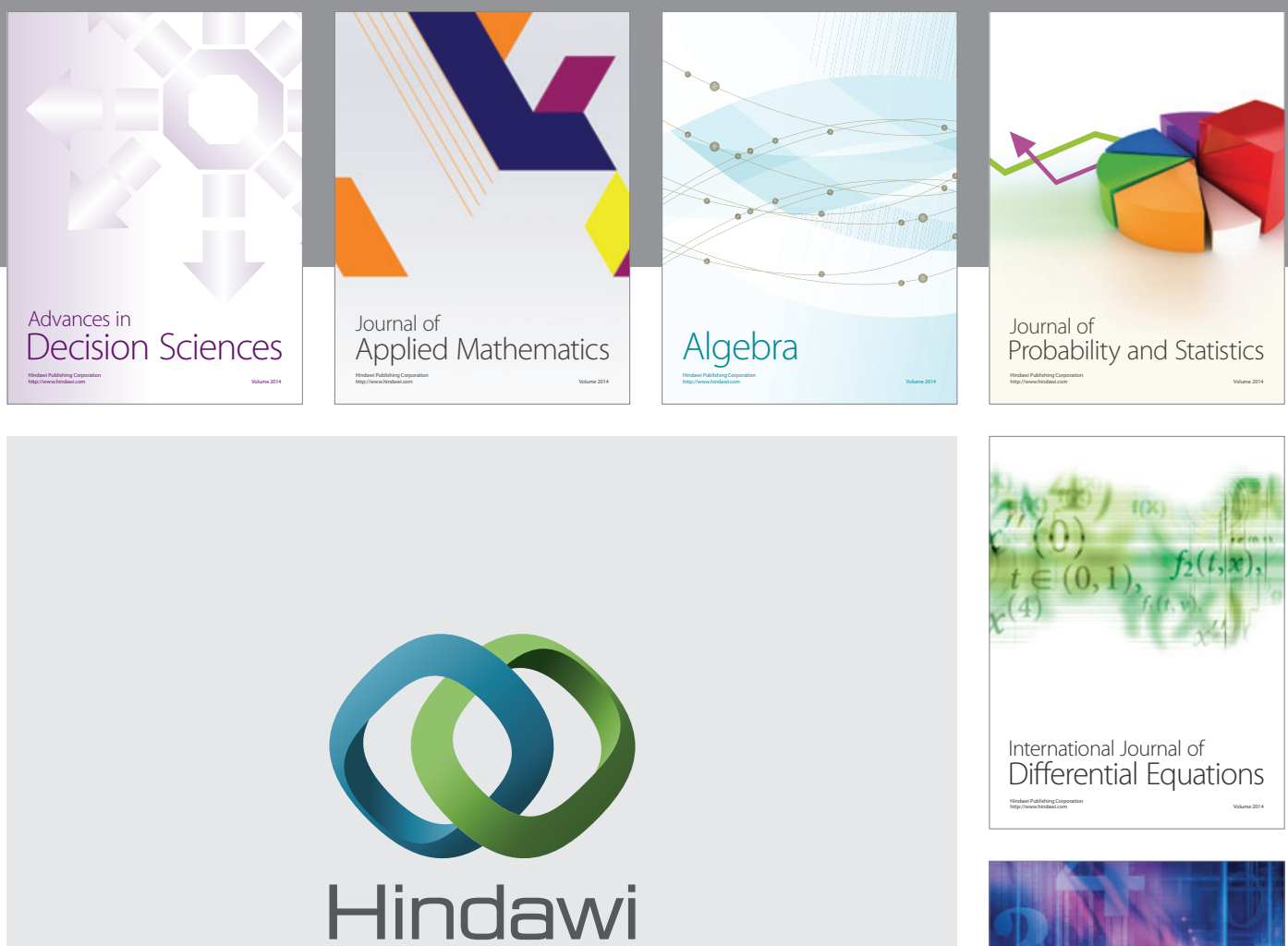

Submit your manuscripts at http://www.hindawi.com
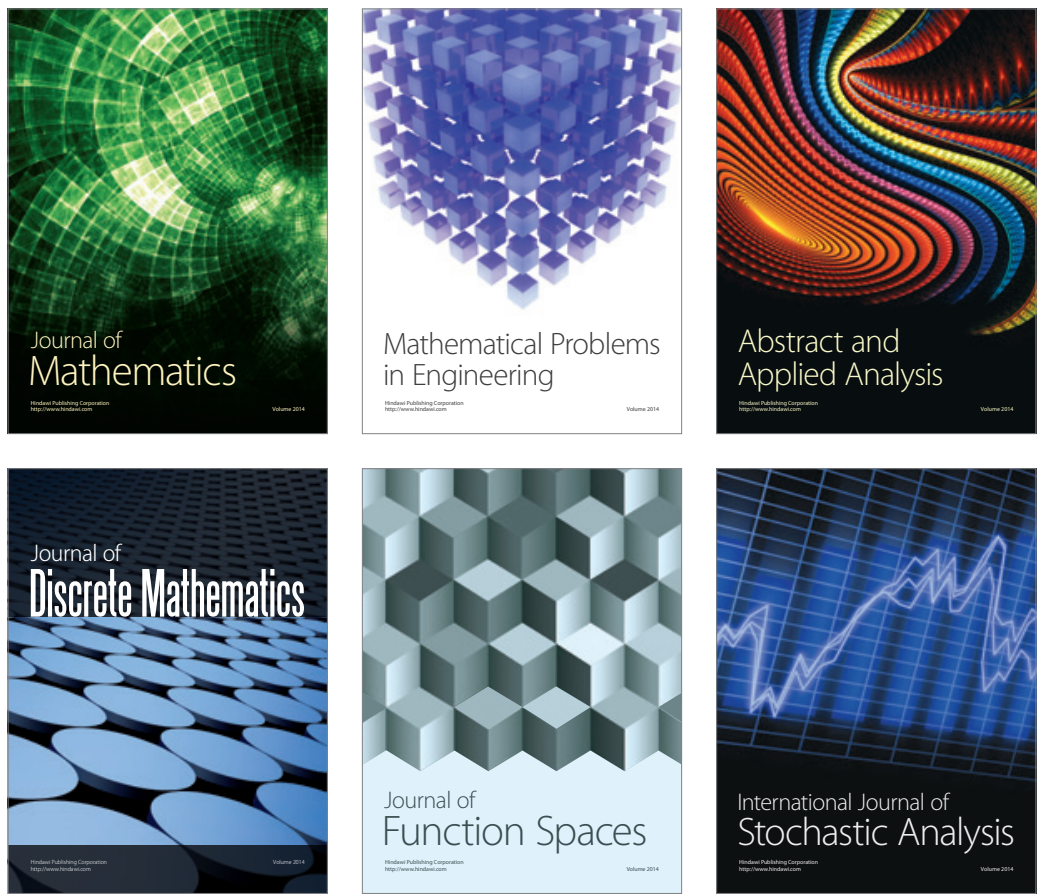

Journal of

Function Spaces

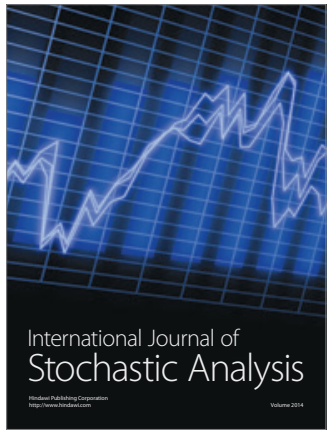

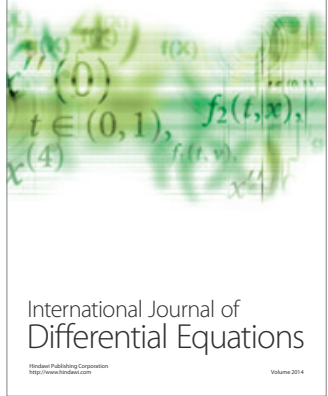
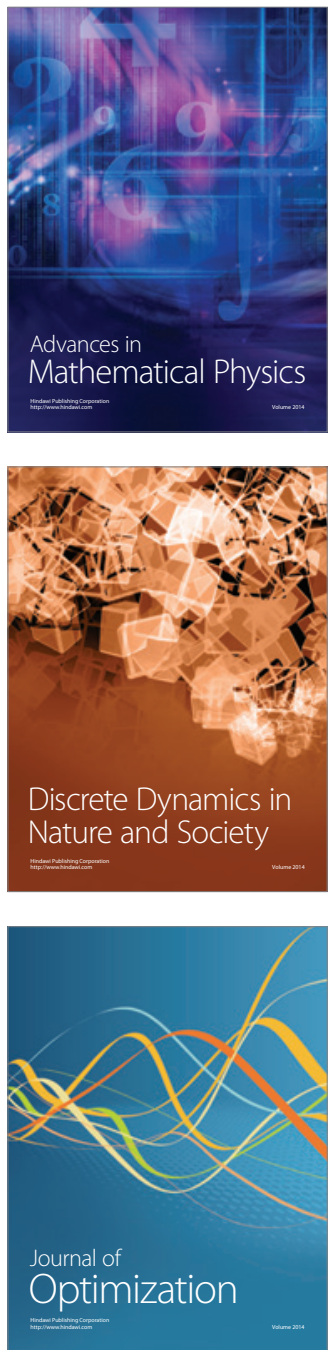